Research Paper

\title{
Osthole Promotes Bone Fracture Healing through Activation of BMP Signaling in Chondrocytes
}

\author{
Pinger Wang1\#, Jun Ying,,2\#, Cheng Luo ${ }^{1,2}$, Xing Jin¹,3, Shanxing Zhang, \\ $\mathrm{Mi}^{5}$, Di Chen ${ }^{6}$, Peijian Tong ${ }^{4}$, Hongting Jin ${ }^{1,4 \bowtie}$ \\ 1. Institute of Orthopaedics and Traumatology, the First Affiliated Hospital of Zhejiang Chinese Medical University, Hangzhou 310053, Zhejiang Province, \\ China \\ 2. First Clinical College of Zhejiang Chinese Medical University, Hangzhou 310053, Zhejiang Province, China \\ 3. Department of Orthopaedics and Traumatology, Wangiiang Sub-District Community Health Service Centre, Hangzhou 310016, Zhejiang Province, China \\ 4. Department of Orthopaedic Surgery, the First Affiliated Hospital of Zhejiang Chinese Medical University, Hangzhou 310006, Zhejiang Province, China \\ 5. Department of Traumatology, Beijing Jishuitan Hospital, 100035, Beijing, China \\ 6. Department of Biochemistry, Rush University Medical Center, Chicago, IL 60612, USA \\ \# These authors contributed equally to this work.
}

$\square$ Corresponding authors: Hongting Jin, M.D. Ph.D., Institute of Orthopaedics and Traumatology, the First Affiliated Hospital of Zhejiang Chinese Medical University, No.548, Binwen Road, Hangzhou, 310053, Zhejiang, China. Tel: 011-86 571-86633057; Fax: 011-86 571-86613684; Email: [hongtingjin@163.com] or Peijian Tong, M.D., The First Affiliated Hospital of Zhejiang Chinese Medical University, No.54, You Dian Road, Hangzhou 310006, Zhejiang, China. Tel: 011-86 571-86613684; Fax: 011-86 571-86613684; Email: [peijiantongzjtcm@163.com]

(c) Ivyspring International Publisher. This is an open access article distributed under the terms of the Creative Commons Attribution (CC BY-NC) license (https://creativecommons.org/licenses/by-nc/4.0/). See http://ivyspring.com/terms for full terms and conditions.

Received: 2017.03.08; Accepted: 2017.04.18; Published: 2017.07.18

\begin{abstract}
Osthole is a bioactive coumarin derivative and has been reported to be able to enhance bone formation and improve fracture healing. However, the molecular mechanism of Osthole in bone fracture healing has not been fully defined. In this study we determined if Osthole enhances bone fracture healing through activation of BMP2 signaling in mice. We performed unilateral open transverse tibial fracture procedure in 10-week-old C57BL/6 mice which were treated with or without Osthole. Our previous studies demonstrated that chondrocyte BMP signaling is required for bone fracture healing, in this study we also performed tibial fracture procedure in Cre-negative and Col2-Cre;Bmp2 flox/flox conditional knockout $(\mathrm{KO})$ mice $\left(\mathrm{Bmp2}^{\mathrm{Col} 2 \mathrm{Cre}}\right)$ to determine if Osthole enhances fracture healing in a BMP2-dependent manner. Fracture callus tissues were collected and analyzed by X-ray, micro-CT ( $\mu \mathrm{CT})$, histology, histomorphometry, immunohistochemistry (IHC), biomechanical testing and quantitative gene expression analysis. In addition, mouse chondrogenic ATDC5 cells were cultured with or without Osthole and the expression levels of chondrogenic marker genes were examined. The results demonstrated that Osthole promotes bone fracture healing in wild-type (WT) or Cre- control mice. In contrast, Osthole failed to promote bone fracture healing in $\mathrm{Bmp}^{\mathrm{CO} / 2 \mathrm{Cre}}$ conditional $\mathrm{KO}$ mice. In the mice receiving Osthole treatment, expression of cartilage marker genes was significantly increased. We conclude that Osthole could promote bone strength and enhance fracture healing by activation of BMP2 signaling. Osthole may be used as an alternative approach in the orthopaedic clinic for the treatment of fracture healing.

Key words: Osthole; Fracture healing; BMP Signaling; Chondrocyte; Endochondral bone formation
\end{abstract}

\section{Introduction}

Bone fractures are one of the most common traumatic injuries in humans [1], especially among elders with advanced osteoporosis [2]. The time length of the fracture healing is an important factor in determining a patient's recovery rate and treatment cost [3]. Bone fracture heals normally in most patients; however, an overall 5 to $10 \%$ of bone fractures result in hypertrophic callus formation and delayed healing process [4].

Clinically, the fracture healing process is determined by patients' health conditions, anatomic locations, as well as quality of the surrounding soft 
tissues. Temporary immobilization and surgical fixation are considered as the treatment choice for most fractures [5]. Unfortunately, many patients refused to undergo invasive operation due to the risk of non-union and delayed union [6]. There is a critical need to develop alternative strategies to promote fracture healing and repair.

Osthole (7-methoxy-8-isopentenoxycoumarin, C15H16O3, $244.39 \mathrm{Da})$ is a bioactive coumarin derivative [7-9] and has been used clinically in the treatment of kidney diseases [10]. Recently, several reports demonstrate that Osthole could prevent bone loss and promoting bone formation [11-13].

Fracture healing process is a specialized repair process that recapitulates aspects of embryonic skeletal development [14, 15]. During the healing process, both periosteal and endochondral ossification processes are involved in new bone formation and the latter process plays a more important role during fracture healing. In addition, multiple growth factor signaling pathways may be activated during fracture healing process [16, 17]. Among them, bone morphogenetic proteins (BMPs) play a more important role in fracture healing process. Recent studies suggest that BMP pathway is associated with the early phase in Osthole-induced osteoblast differentiation [18, 19]. In addition, Osthole induces endochondral ossification by up-regulation of mature osteoblast marker genes [20]. However, the underlying mechanism of Osthole-induced chondrocyte differentiation during fracture healing was not well characterized. Our current study aimed to investigate the in vivo effects and the molecular mechanisms by which Osthole promotes bone fracture healing.

\section{Methods and Materials}

\section{Preparation of Osthole}

Osthole (No. 110822-200305, purity > 98\%, $244.39 \mathrm{Da})$ (Figure 1A) was purchased from the National Institute for the Control of Pharmaceutical and Biological products (Beijing, China) and was dissolved in dimethylsulfoxide (DMSO) (less than $0.1 \%)$.

\section{Experimental Animals}

10-week-old male C57BL/ 6 mice were obtained from the animal center of the Zhejiang Chinese Medical University (Y2111184). They were divided into two groups: Osthole treatment group $(30 \mathrm{mg} / \mathrm{kg}$, local subcutaneous injection, daily for 28 days) [11] and vehicle control (PBS) group.

To generate chondrocyte specific Bmp2 conditional KO mice, $B m p 2^{\text {floxfflox }}$ mice (obtained from Dr. Di Chen, Rush University, Chicago, USA) [21, 22] were bred with Col2-Cre transgenic mice. The Col2-Cre transgenic mice could efficiently target growth plate chondrocytes [23, 24]. All mice were under C57BL/6 background and mouse genotyping was determined by PCR using DNA extracted from tail biopsy tissues. PCR primer sequences for genotyping were as follows, MP1-600 F (A) 5'-AGGGTTTCAGGTCA GTTTCCG, MP1-Hap-rv2 (B) 5'-GATGATGAGG TTCTTGGCGG, and MP1-1615RW (C) 5'-TCCGA AGGTAAGTGTGCTTGG. Cre-positive and $B m p 2^{f l o x}$ flox male littermates were chosen as experimental groups while Cre-negative male littermates were used as controls (mean body weight $22 \pm 2 \mathrm{~g}$ ). All mice had free access to food and water during the entire study.

\section{Tibial Fracture Model}

In this study, a unilateral (right side) open transverse tibial fracture with intramedullary needle fixation was selected as the bone fracture model in 10-week-old C57BL/6, Cre-negative and Bmp2Col2Cre mice $[22,25,26]$. Anesthesia was induced with Ketamine $(60 \mathrm{mg} / \mathrm{Kg})$ via intraperitoneal injection. Next a $1.5 \mathrm{~cm}$-long incision was made along the anterior-medial surface and a 26 gauge syringe needle was inserted into the bone marrow cavity of the tibia through the tibial plateau on the medial side of the patellar ligament. Then the needle was removed, and a No. 11 surgical blade was used to transect the diaphysis of the tibia at the midpoint. A 26 gauge needle was then inserted into the tibia to stabilize the fracture. A 4-0 silk suture was used to close the wound and pain was managed using buprenorphine (in drinking water) for the first three days after surgery [26].

\section{Radiographic and $\mu \mathrm{CT}$ Analyses}

After operation, X-ray radiographic analysis was performed immediately after fracture to ensure the fracture pattern and the position of the fixation needle. Mice were sacrificed at days 7, 10, 14, 21 and 28 for histological analysis after surgery $(n=10$ in each time point). Fracture healing was examined by the assessment of bridging across cortices. The extent of bridging between the fracture gap was determined in a blinded pattern by three independent investigators following the criteria: 1) grade 1: no healing (no indication or only some indications mineralized bridging); 2) grade 2: partial healing (more consolidated bridging or almost completely mineralized bridging); and 3) grade 3: complete healing (completely mineralized bridging). Specimens were scanned at 10.5-micron isotropic resolution using a micro-computed tomography $(\mu \mathrm{CT})$ (Skyscan 1176; Bruker $\mu \mathrm{CT}$, Kontich, Belgium) at each time points $[26,27]$. Callus total volume (TV), callus bone 
volume (BV), callus mineralized volume fraction $(\mathrm{BV} / \mathrm{TV})(\%)$ and callus bone mineral density (BMD) were determined ( $\mathrm{n}=10$ in each time point).

\section{Biomechanical Testing}

Tibia bone samples were harvested and surrounding soft tissues were carefully removed $(\mathrm{n}=10$ at days 10,14, 21, and 28). Samples were fixed in aluminum square tubes $(0.5 \mathrm{~cm})$ filled with bone cement to make sure that fracture lines were in the middle of the interval. Specimens were mounted on an EnduraTec TestBenchTM system with a 200 N.mm torque cell (EnduraTec TestBenchTM system, Bose Corp., Minnetonka, MN) and tested in torsion at a rate of 10 /sec until failure to determine maximum torque, stiffness and toughness of fracture callus [22].

\section{Histology, Histomorphometry and Immunohistochemistry (IHC)}

Bone samples were harvested ( $\mathrm{n}=10$ at days 7,10 , 14, 21 and 28) and fixed in 10\% normal buffered formaldehyde for 3 days, decalcified in 14\% EDTA solution for 14 days at room temperature and then embedded in paraffin. Tissue sections at the fracture site were cut longitudinally at thickness of 3- $\mu \mathrm{m}$ and prepared for Alcian blue/H\&E staining and TRAP staining (day 14). Histomorphometric analysis $(n=10$ in each time point) was conducted using OsteoMetrics software (Decatur, GA). The mineralized volume of the cortices, the area of the periosteal calluses, and the mineralized and cartilaginous volume of the calluses were measured [22, 26]. The numbers of TRAP-positive multinucleated osteoclasts and percentage of osteoclast surface of the calluses were also measured. IHC was performed using anti-type II collagen (NeoMarkers, Inc. Fremont, CA, USA) and anti-pSmad1/5/8 (Santa Cruz Biotechnology, Santa Cruz, CA, USA) antibodies on the $3-\mu \mathrm{m}$ thick tissue sections (days 7).

\section{Quantitative Gene Expression Analysis}

Total RNA was extracted from the callus tissue including adjacent bone on either side of the fracture line $(1 \mathrm{~mm})$ using the PureLink ${ }^{\mathrm{TM}}$ RNA Mini Kit (Invitrogen, Carlsbad, CA, USA) ( $\mathrm{n}=6$ in each time point). One microgram total RNA was used to synthesize cDNA using iScripts cDNA Synthesis Kit (Bio-Rad, Hercules, CA, USA). Real-time PCR amplification was performed using murine gene specific primers and SYBR green real-time PCR kit (Bio-Rad, Hercules, CA, USA). The levels of the target gene expression were normalized to that of $\beta$-actin. Expression levels of chondrogenic (Sox9, Col2a1, Col10a1) and osteogenic (Runx2, osteocalcin) marker genes was examined. Forward and reverse primers specific for the genes listed in Table 1.
Table 1. Primer name and sequences for PCR analysis

\begin{tabular}{ll}
\hline Primer Name & Sequences \\
\hline$\beta$-Actin forward & 5'-GGAGATTACTGCCCTGGCTCCTA-3' \\
$\beta$-Actin reverse & 5'-GACTCATCGTACTCCTGCTTGCTG-3' \\
Sox 9 forward & 5'-GAGGCCACGGAACAGACTCA-3' \\
Sox 9 reverse & 5'-CAGCGCCTTGAAGATAGCATT-3' \\
Col2a1 forward & 5'-TGGTCCTCTGGGCATCTCAGGC-3' \\
Col2a1 reverse & 5'-GGTGAACCTGCTGTTGCCCTCA-3' \\
Col10a1 forward & 5'-ACCCCAAGGACCTAAAGGAA-3' \\
Col10a1 reverse & 5'-CCCCAGGATACCCTGTTTTT-3' \\
Runx2 forward & 5'-GAGGGCACAAGTTCTATCTGGA-3' \\
Runx2 reverse & 5'-GGTGGTCCGCGATGATCTC-3' \\
Osteocalcin forward & 5'-AGGGAGGATCAAGTCCCG -3' \\
Osteocalcin reverse & 5'-GAACAGACTCCGGCGCTA-3' \\
\hline
\end{tabular}

\section{Cell Culture}

Mouse chondrogenic ATDC5 cells (RIKEN Cell Bank, Tsukuba, Japan) were cultured in maintenance medium containing 1:1 Dulbecco's modified Eagle medium (DMEM)/F-12 medium (Sigma, St. Louis, MO, USA), supplemented with $5 \%$ fetal bovine serum (FBS), $100 \mathrm{U} / \mathrm{mL}$ penicillin, $100 \mathrm{mg} / \mathrm{mL}$ streptomycin (Gibco, Grand Island, NY, USA), 1\% L-glutamine (Gibco). After reaching 100\% confluent, cells were centrifuged and then were plated in 12-well plates (Corning, NY, USA) at the center of each well for 2-3 $\mathrm{h}$ before adding chondrogenic differentiation medium (maintenance medium plus $10 \mu \mathrm{g} / \mathrm{mL}$ insulin, 10 $\mu \mathrm{g} / \mathrm{ml}$ transferrin and $30 \mathrm{nM}$ sodium selenite). All cells were maintained at $37^{\circ} \mathrm{C}$ in a humidified $5 \% \mathrm{CO}_{2}$ incubator. The medium was changed every other day. After 2 days of culture, cells were treated with Osthole at concentrations of $0,10,50,100 \mu \mathrm{M}$ for one week (6 wells per group) [11]. Then total RNA was extracted and the levels of chondrogenic associated genes (Sox9, Col2a1, Col10) were analyzed.

\section{Statistical Analysis}

All values were presented as mean \pm standard deviation. Statistical analyses included unpaired Student's $t$-tests and one-way ANOVA test followed by the Tukey-Kramer test. The statistical tests were performed using the software SPSS 17.0. $P<0.05$ was considered as significant.

\section{Results}

\section{Osthole Promotes Bone Fracture Healing in Mice}

Osthole (7-methoxy-8-isopentenoxycoumarin, C15H16O3, 244.39 Da) (Figure 1A) is an O-methylated coumarin isolated from plants such as Cnidium monnieri, Angelica archangelica and Angelica pubescens [29]. In the present studies, we examined the effect of Osthole on bone fracture healing. The results of radiographic analysis showed that administration of Osthole enhanced bone callus 
formation. Compared to the PBS control group, the presence of a fracture gap was blurry in Osthole treated group at days 10, 14 and 21 (Figure 1B and Table 2).

Table 2. Radiographic analysis of fracture gaps

\begin{tabular}{lllllll}
\hline & \multicolumn{3}{l}{ PBS Control $(\mathrm{n}=10)$} & \multicolumn{3}{l}{ Osthole $(\mathrm{n}=10)$} \\
\hline Healing & No & Partial & Complete & No & Partial & Complete \\
$\mathrm{d} 7$ & 10 & 0 & 0 & 9 & 1 & 0 \\
$\mathrm{~d} 10$ & 7 & 3 & 0 & 5 & 5 & 0 \\
$\mathrm{~d} 14$ & 2 & 8 & 0 & 0 & 4 & 6 \\
$\mathrm{~d} 21$ & 0 & 6 & 4 & 0 & 0 & 10 \\
\hline
\end{tabular}

Moreover, there was almost no fracture gap visible at day 28 in Osthole treated group, thus further confirming the beneficial role of Osthole on the normal fracture healing process (Figure 1B). In contrast the control group showed obvious fracture gap at the same time point. $\mathrm{\mu CT}$ data showed that the bone volume in Osthole-treated mice was increased compared to PBS control group at days 7, 10 and 14 (Figure 2A-C), supporting the radiographic findings. Results of biomechanical testing showed that treatment with Osthole enhanced bone mechanical strength (Figure 2D-F). The maximum torque and stiffness were significantly increased at days 10 and 14 in Osthole treated group compared to controls (Figure 2D and E).

Histologic and histomorphometric analyses showed that the progression of fracture healing was accelerated in Osthole-treated group. Cartilage area was determined by Alcian blue staining, which is significantly enlarged by day 7 in Osthole-treated mice (Figure $3 \mathrm{~A}$ and $\mathrm{B}$ ). The woven bone area was

B

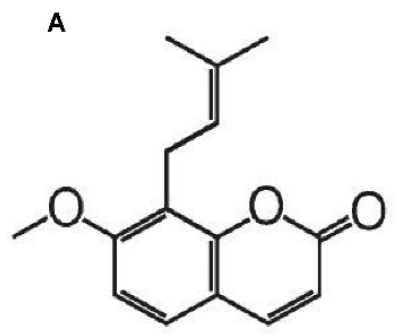

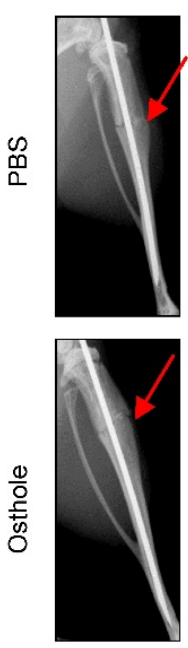

7
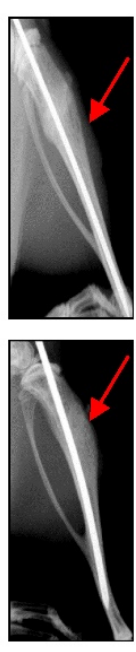

10
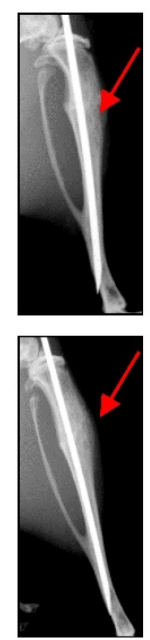

14 increased at days 7, 10 and 14 after surgery in Osthole-treated group (Figure $3 \mathrm{~A}$ and C). The increase in cartilage area was supported by gene expression results showing that expression of the early cartilage marker genes such as Sox 9 and Col2a1 were increased at day 7 in Osthole-treated group (Figure 4A and B). The levels of the later cartilage hypertrophic marker Col10a1 were also increased at day 10 in Osthole-treated mice (Figure 4C). The expression of bone marker genes, Runx2 and osteocalcin $(O C N)$, was increased at day 21 in Osthole-treated group (Figure 4D and E). The changes in expression of cartilage and bone marker genes are consistent with the process and timing of endochondral ossification during the fracture healing process.

\section{Osthole Promotes Bone Fracture Healing through BMP Signaling in Chondrocytes}

Our findings suggest that Osthole can promote bone fracture healing by accelerating endochondral ossification. The expression of the type II collagen protein was further confirmed by IHC staining of the callus at day 7, with increased Col-II expression in Osthole-treated group (Figure 5A). Moreover, increased pSmad1/5/8 expression was detected at day 7 in Osthole-treated group (Figure 5B). Furthermore a mouse chondrogenic ATDC5 cells were used for in vitro experiments. Osthole promoted chondrogenesis in a dose-dependent manner (10-100 $\mu \mathrm{M})$, as shown by the up-regulation of chondrocyte related marker genes such as Sox9, Col2a1 and Col10a1 (Figure 5C).

To determine if Osthole regulates osteoclast formation, we also performed TRAP staining in fracture callus at day $14 . \quad$ No significant changes in osteoclast numbers were found after Osthole treatment (Figure 6A-C), suggesting that Osthole has no significant effect on osteoclast formation during fracture healing.

Figure 1. Osthole promotes bone fracture healing in mice. (A) Chemical structure of Osthole. (B) Fracture procedure was performed in 10 -week-old mice treated with vehicle or Osthole. X-ray radiographic analysis showed clear fracture lines at day 7-21 in mice of control group, and blurry at day 10 and 14 in Osthole treated group, suggesting that fracture healing process is accelerated by Osthole. Red arrows indicate the fracture lines. 

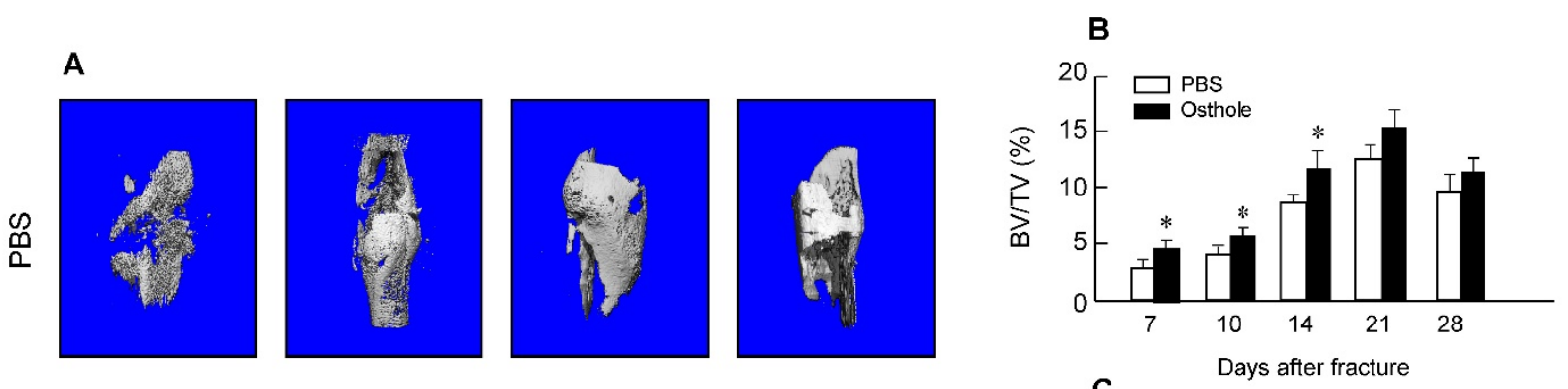

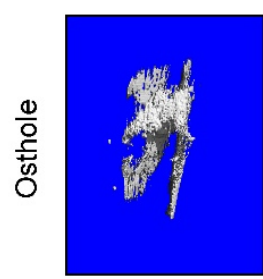

7

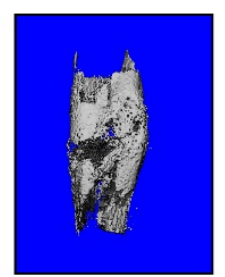

10

D

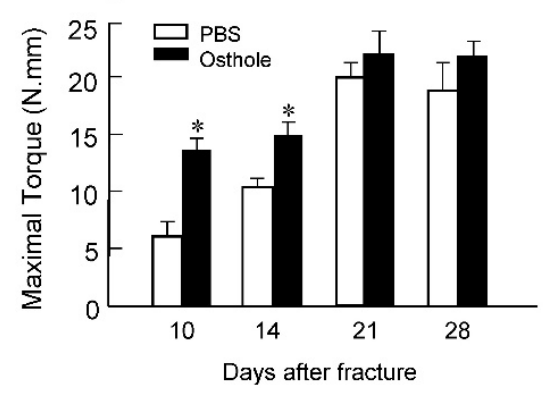

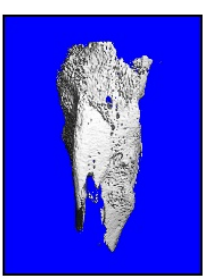

14

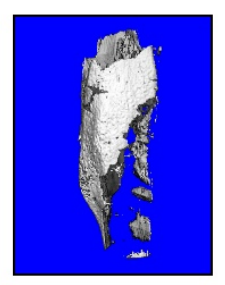

21
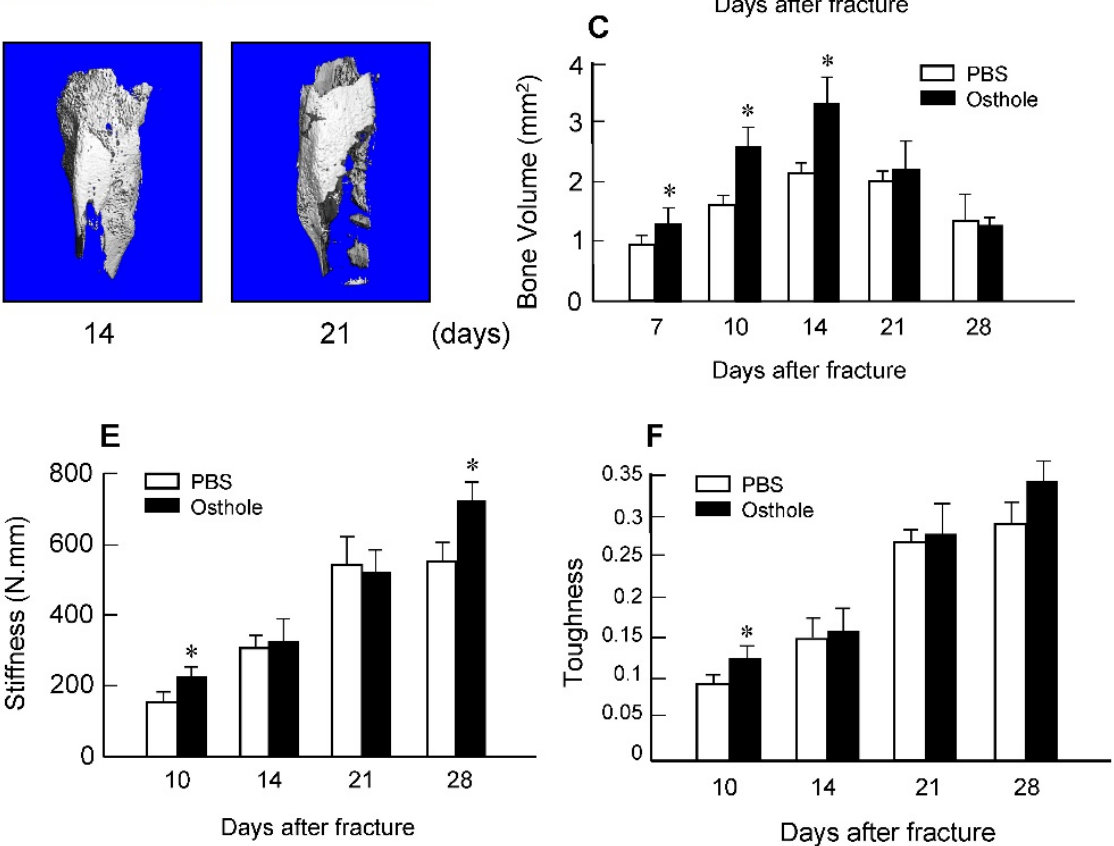

Figure 2. $\mu \mathrm{CT}$ and mechanical testing were performed in bone samples collected from mice 7, 10, 14, 21 and 28 days after surgery. (A-C) $\mu \mathrm{CT}$ analysis indicates that bone volume (\%, BV/TV) was increased in callus tissues 7, 10 and 14 days after surgery in the mice treated with Osthole. (D-F) Mechanical testing showed that maximal torque (day 10 and 14 ) and stiffness (day 10 and 28 ) were significantly increased by the treatment with Osthole. Data are presented as means \pm SD. $* P<0.05, \mathrm{n}=10$.

To provide the definitive evidence about the role of Bmp2 in Osthole-induced fracture healing, we examined the effect of Osthole on fracture healing in chondrocyte-specific Bmp2 conditional $\mathrm{KO}$ mice. Radiographic data demonstrated that treatment with Osthole enhanced bone callus formation in Cre-negative group compared to the Bmp2Col2Cre group. The appearance of a fracture gap was blurry in Cre-negative group at earlier days 10, 14 and 21 than Bmp2Col2Cre group (Figure 7 and Table 3). At day 28, there was no obvious fracture gap in Cre-negative group. In contrast, obvious fracture gaps were seen in Bmp2Col2Cre group. These results indicate the essential role of BMP2 in Osthole-induced bone fracture healing process. $\mu \mathrm{CT}$ analysis demonstrated that the bone volume in Cre-negative control group was increased in response to Osthole treatment compared to Bmp2Col2Cre group at days 7, 10 and 14 (Figure 8A-C). In addition, the mechanical strength of the tibia was significantly decreased in Bmp2 $2^{\mathrm{Col} 2 \mathrm{Cre}}$ group, compared to the Cre-negative group in the presence of Osthole $(P<0.05)$ (Figure 8D). The stiffness also significantly decreased at days 10 and 14 in Bmp2Col2Cre group, compared to Cre-negative control group in the presence of Osthole (Figure 8E). However, no significant effect was found on the stiffness and toughness at day 21 and 28 after Osthole treatment (Figure 8E, F).

Table 3. Radiographic analysis of fracture gap on Cre-negative and Bmp2 2 ol2Cre groups both with Osthole treatment

\begin{tabular}{|c|c|c|c|c|c|c|}
\hline \multicolumn{4}{|c|}{ Cre-negative Control with Osthole $(n=10)$} & \multicolumn{3}{|c|}{ Bmp2Col2Crewith Osthole $(\mathrm{n}=10)$} \\
\hline Healing & No & Partial & Complete & No & Partial & Complete \\
\hline $\mathrm{d} 7$ & 9 & 1 & 0 & 10 & 0 & 0 \\
\hline d10 & 5 & 5 & 0 & 8 & 2 & 0 \\
\hline d14 & 0 & 4 & 6 & 4 & 5 & 1 \\
\hline d21 & 0 & 0 & 10 & 1 & 6 & 3 \\
\hline
\end{tabular}



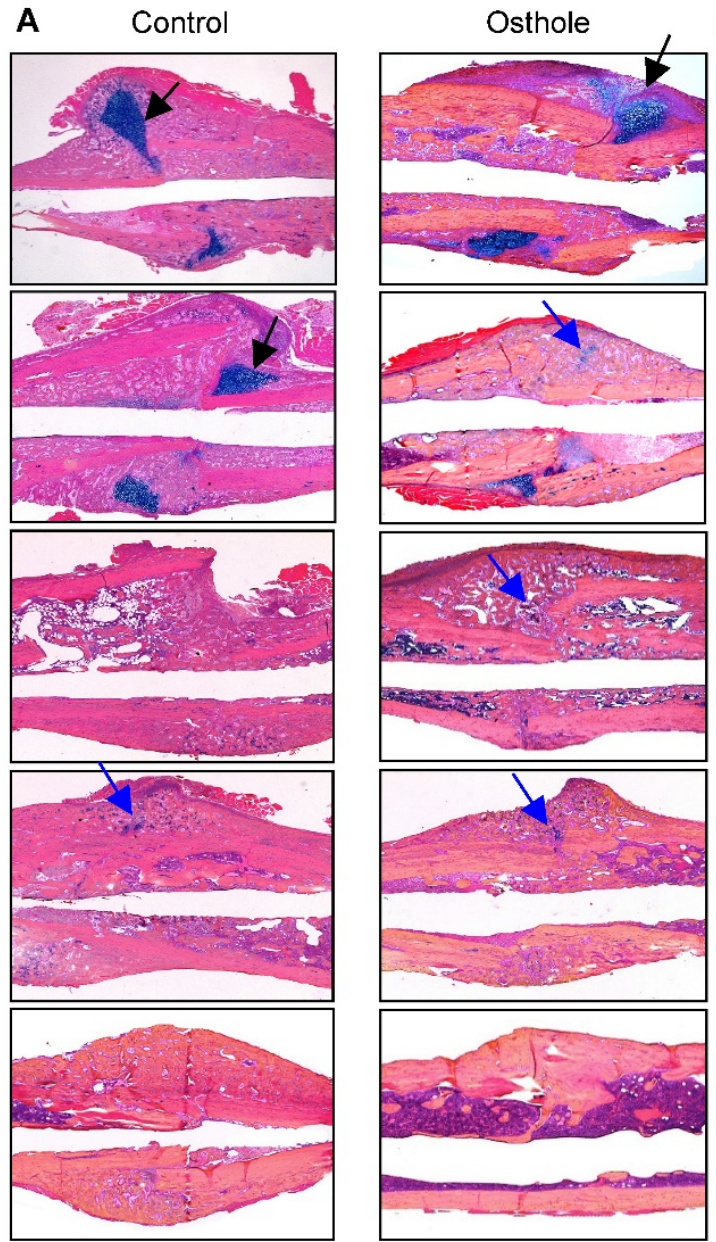

(days)

7

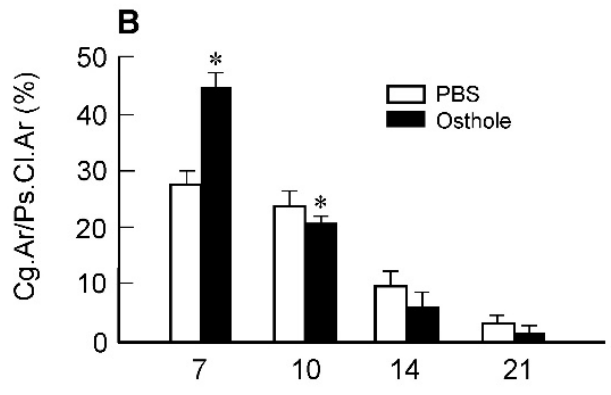

14

Days after fracture

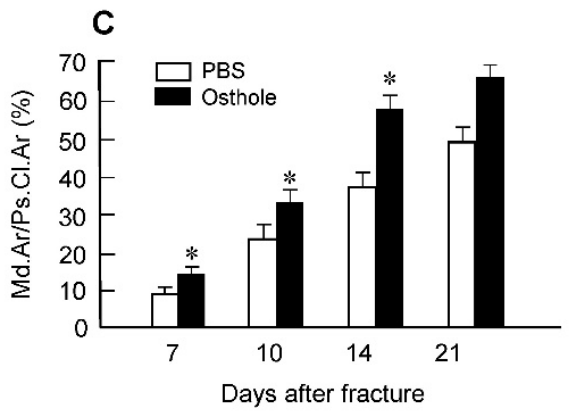

Figure 3. Osthole increases cartilage and bone volume of fracture callus. (A) Histological analysis showed that Osthole accelerated the fracture healing process. (B) The Alcian blue staining positive cartilage area of callus tissues, (\%, Cg.Ar/Ps.Cl.Ar) was increased after Osthole treatment at day 7 and slightly reduced at day 10. (C) New woven bone formation in callus tissues (\%, Md.Ar/Ps.Cl.Ar) was enhanced by Osthole treatment at day 10-21. Black arrows: cartilage area; blue arrows: woven bone area. Data are presented as means $\pm S D$. $* P<0.05, n=10$.
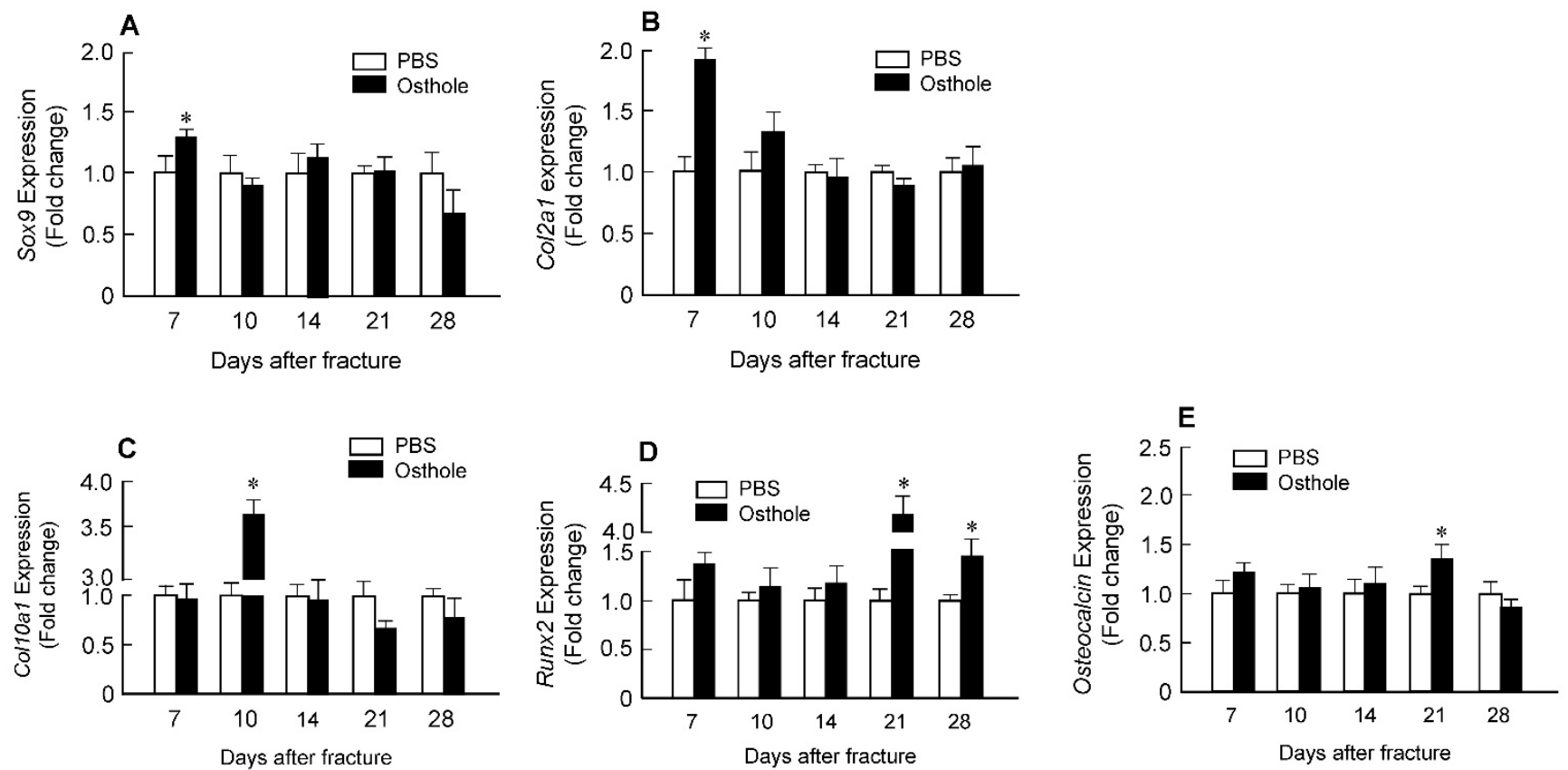

Figure 4. Osthole up-regulates expression of chondrocyte- and osteoblast-specific marker genes in callus tissues. Total RNA was extracted from callus tissues at day $7,10,14$, 21 and 28 in the mice treated with or without Osthole. (A-C) Expression of Sox 9 and Col2al was significantly increased at day 7; and expression of CollOal was significantly increased at day 10. (D, E) Expression of Runx2 was significantly increased at day 21 and 28, and expression of osteocalcin (OC) was significantly increased at day 21 . Data are presented as means \pm SD. $* P<0.05, n=6$. 
A Control

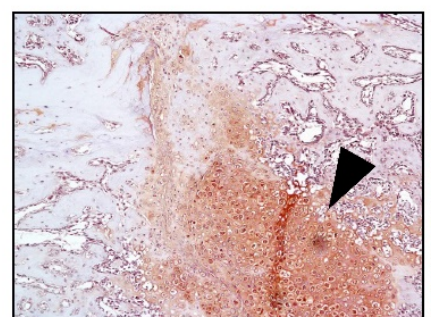

type II collegen (day 7)

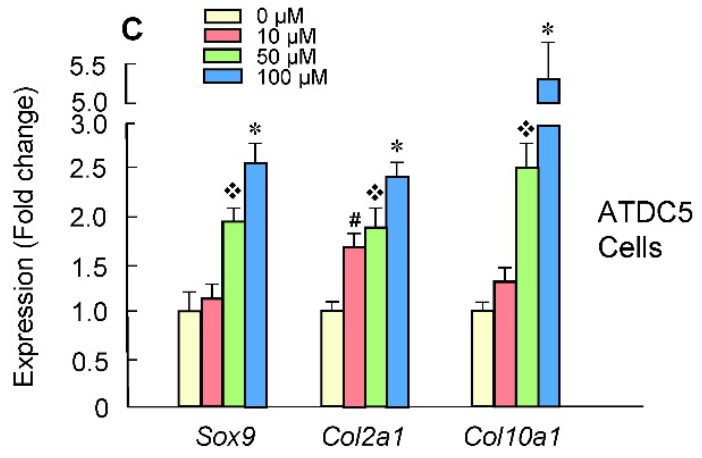

IgG control
B

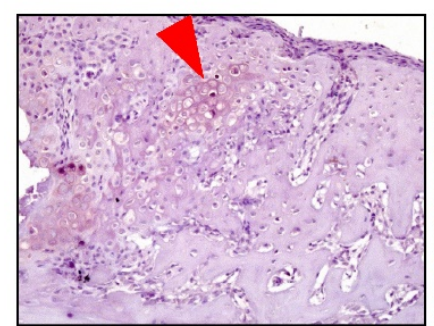

Osthole

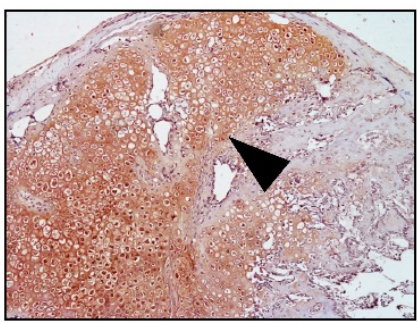

Osthole

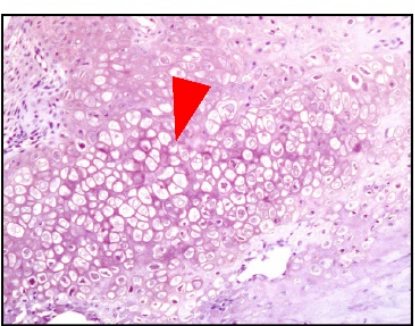

pSmad1/5/8 (day 7)

Figure 5. Osthole promotes bone fracture healing through activation of BMP signaling in Chondrocytes. (A, B) Type II collagen protein and pSmad1/5/8 levels were significantly increased in Osthole treatment group (day 7). Black arrows: type II collagen-positive cells, and red arrows: pSmad1/5/8-positive cells. (C) A mouse chondrogenic cell line, ATDC5, was used to confirm the in vivo data. Osthole up-regulates chondrogenesis related genes, such as Sox 9 , Col2al and CollOal, especially at the concentration of $100 \mu \mathrm{M}$. Data are presented as means \pm SD. $\# P<0.05,10 \mu \mathrm{M}$ compared with $0 \mu \mathrm{M}$; ${ }^{*} \mathrm{P}<0.05,50 \mu \mathrm{M}$ compared with $0 \mu \mathrm{M}$; and $* P<0.05,100 \mu \mathrm{M}$ compared with $0 \mu \mathrm{M}$.

\section{A}

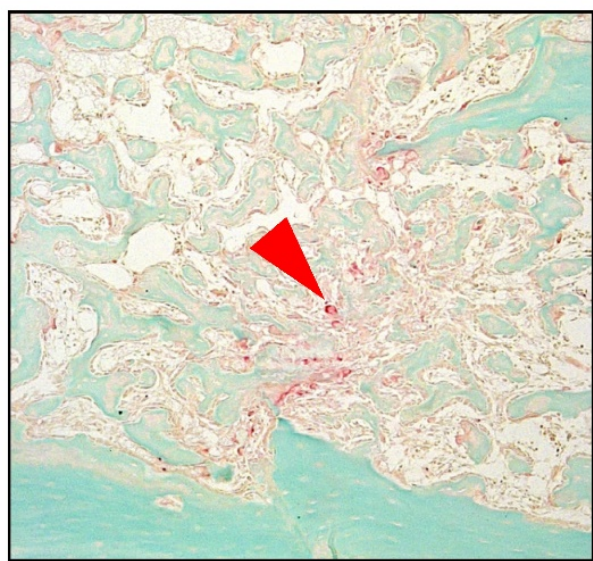

Osthole

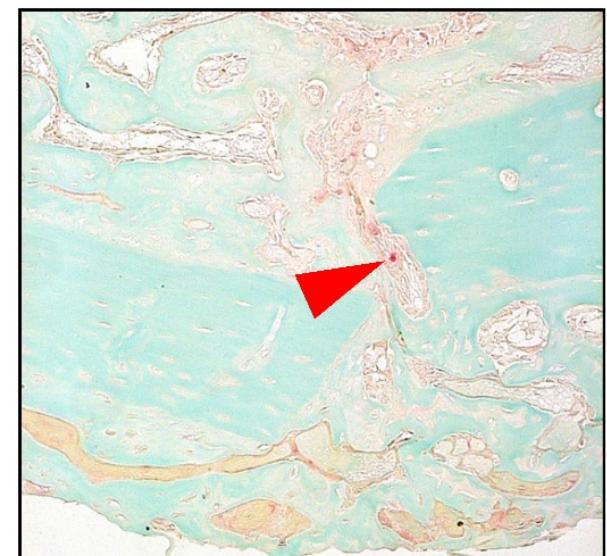

Day 14
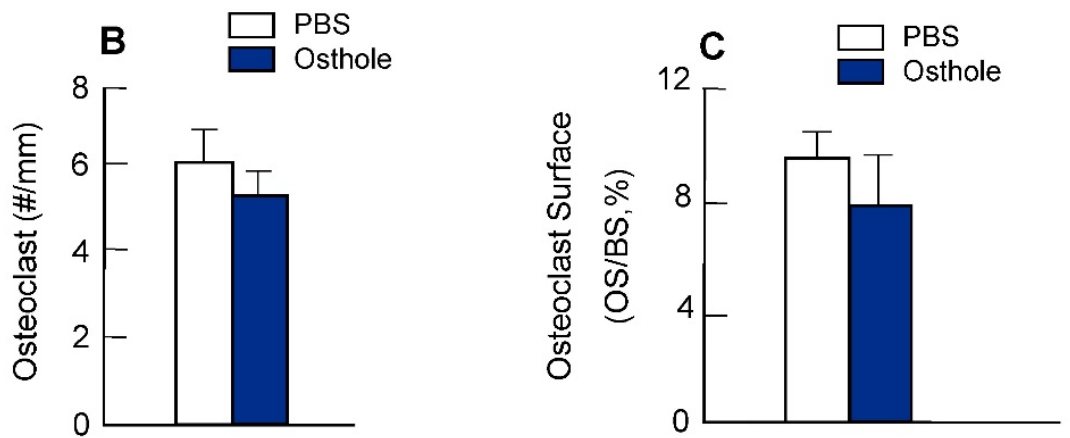

Figure 6. Osthole had no significant effect on osteoclast formation during fracture healing. (A-C) TRAP staining was performed on day 14 callus tissues and showed no significant difference in osteoclast numbers and osteoclast surface percentage after Osthole treatment. Purple arrows: TRAP-positive multiple nuclear osteoclasts. Data are presented as means $\pm S D, n=6$. 

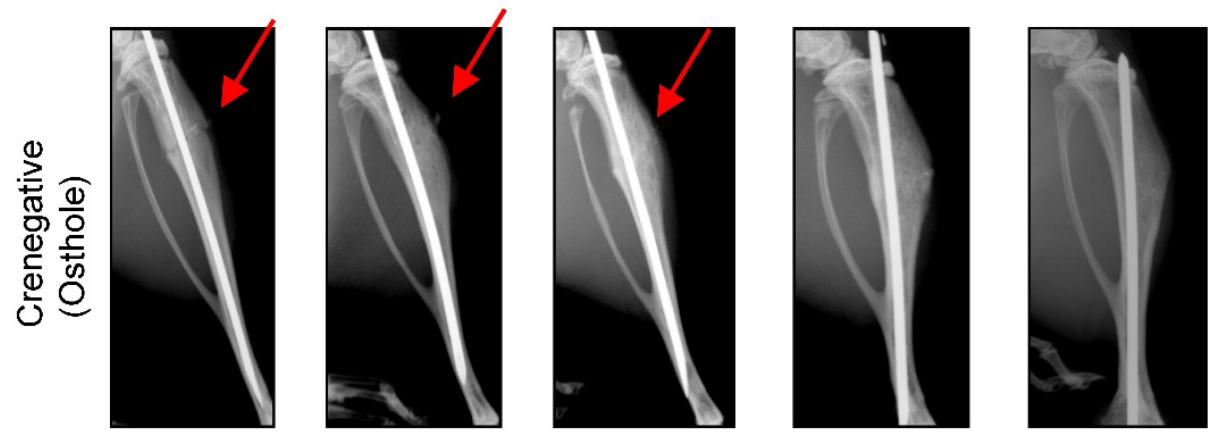

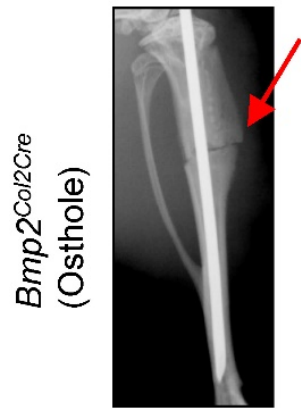

7

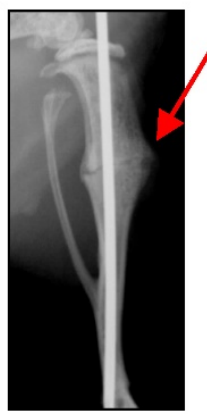

10

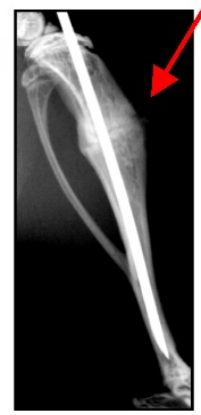

14

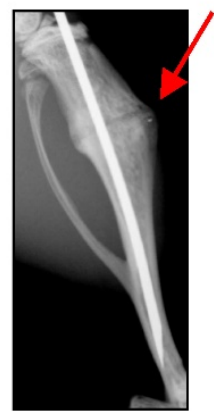

21

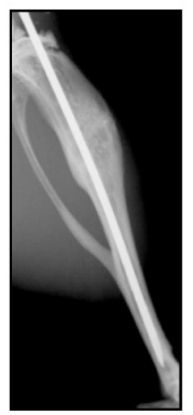

28

(days)

Figure 7. Osthole accelerated bone fracture healing in Cre-negative group compared with $B m p 2$ Col2cre group. X-ray radiographic analysis was performed on mice $7,10,14,21$ and 28 days after surgery. Clear fracture lines were found on radiographs at day 7-21 in Bmp2 ${ }^{C o l 2 C r e}$ group, and were indistinguishable at day 10 and 14 in Cre-negative group. These data suggest that $B m p 2$ is required for Osthole to promote bone fracture healing. Red arrows indicate the fracture lines.
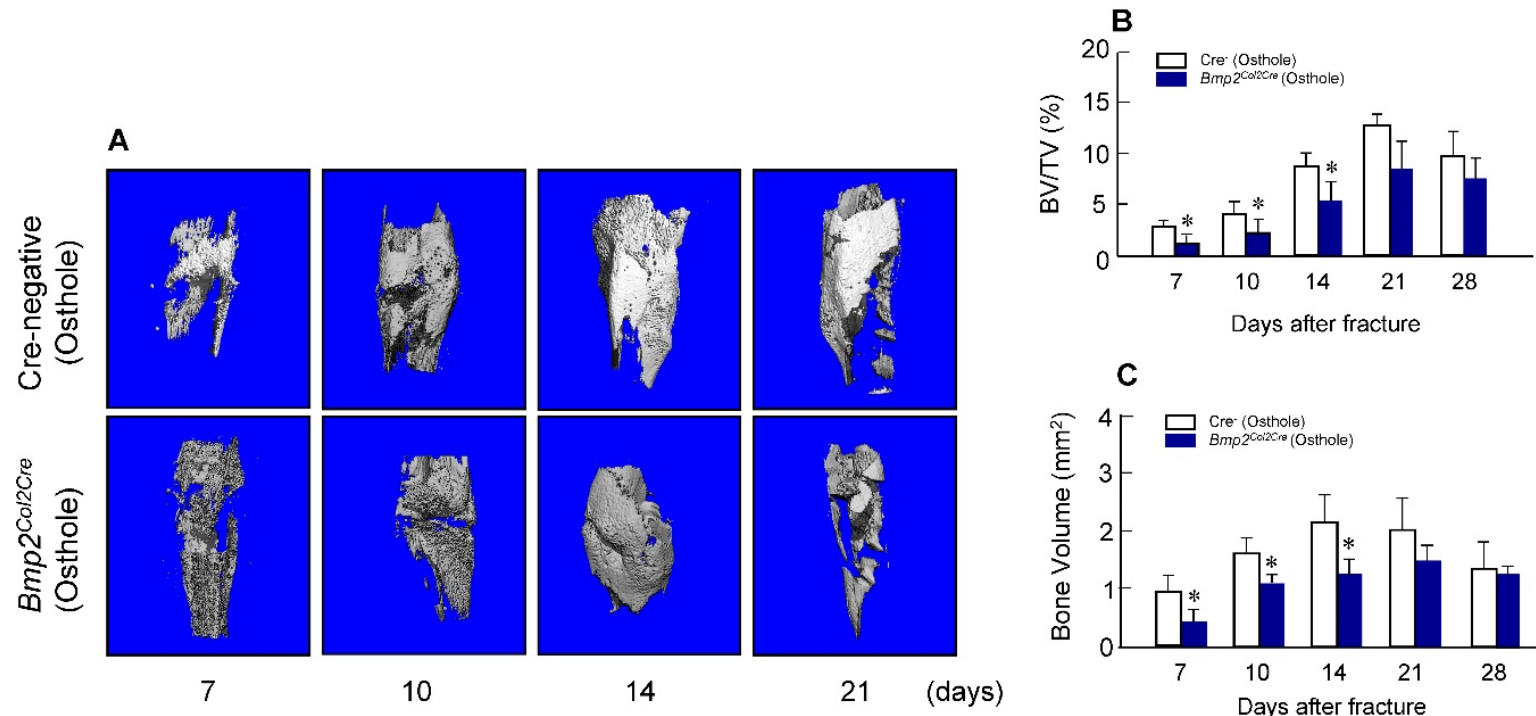

C

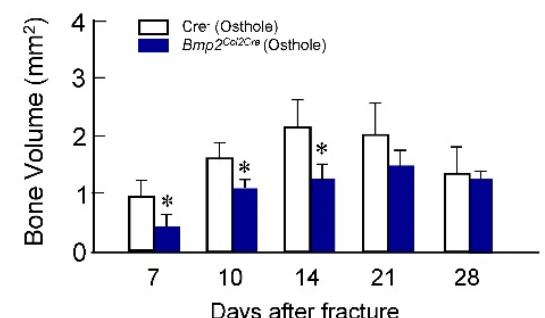

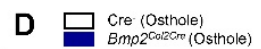
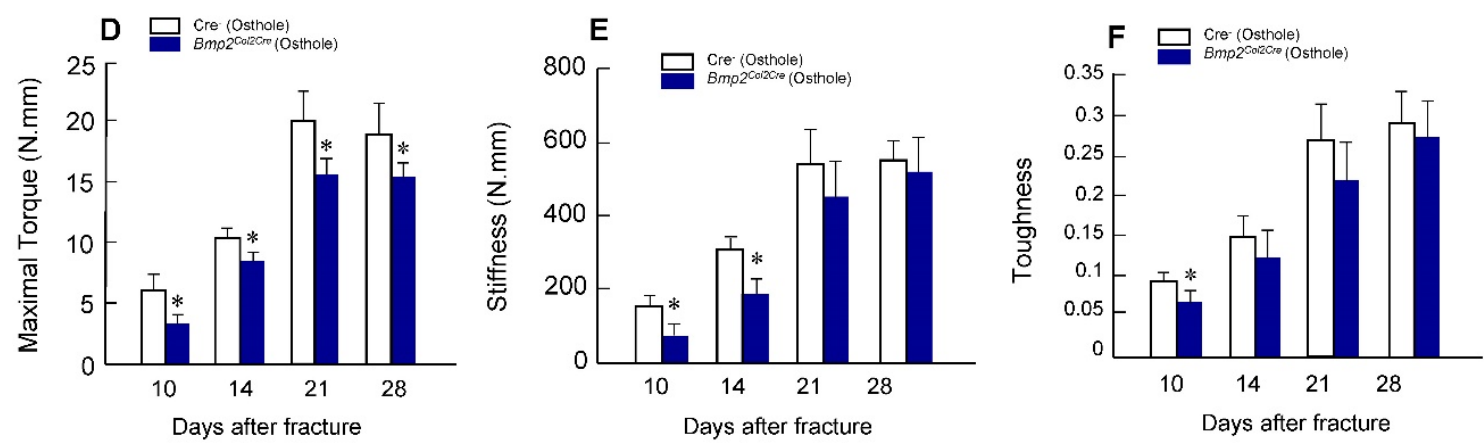

Figure 8. Osthole-induced increases in bone volume and mechanical strength were inhibited in Bmp2 Col2Cre KO mice. $\mu C T$ analysis (A-C) and mechanical testing (D-F) were performed in Cre-negative and $\mathrm{Bmp2Col2Cre} \mathrm{KO}$ mice 7, 10, 14 and 21 days after fracture procedure. Both Cre-negative and $\mathrm{Bmp2Col2Cre} \mathrm{KO}$ mice were treated with Osthole. Bone volume and BV/TV (\%) were significantly increased in callus tissues at day 7,10 and 14 in Cre-negative mice treated with Osthole. In contrast, Osthole-induced increase in bone volume was inhibited in Bmp2Col2cre KO mice (B, C). The effects of Osthole on maximal torque (day 10-28), stiffness (day 10 and 14), and toughness (day 10) were also inhibited in $B m p 2$ Col2Cre group (D-F). Data are presented as means $\pm S D$. $* P<0.05, n=10$. 
Alcian blue/H\&E stained sections from Cre-negative group and $\mathrm{Bmp} 2^{\mathrm{Col} 2 \mathrm{Cre}}$ group, both treated with Osthole, at day 7, 10, 14, 21 and 28, highlighted the characteristic differences in cartilage area of callus tissues and woven bone areas. Results of histologic and histomorphometric analyses revealed that fracture healing progress was delayed in Bmp2Col2Cre group. At day 7, cartilage area was significantly enlarged in $\mathrm{Cre}^{-}$control group after Osthole treatment (Figure 9A and B). And woven bone area was increased at day 10 after surgery in Crecontrol group (Figure 9A and C). To further determine the role of BMP2 in Osthole-enhanced bone fracture healing, we analyzed the gene expression as well. Our results revealed that treatment with Osthole resulted in an increase in the expression of the early cartilage marker genes, such as Sox9 and Col2a1, at day 7 and the later cartilage hypertrophic marker Col10a1 at day 10 in Cre- control mice (Figure 10A-C). The expression of bone marker Runx2 was initially increased at day 10 in Cre- control group (Figure 10D). In addition, Osthole did not affect the expression of bone marker OCN (Figure 10E). These results suggest that Osthole promotes bone fracture healing mainly through activation of BMP signaling during cartilage formation in callus tissue.

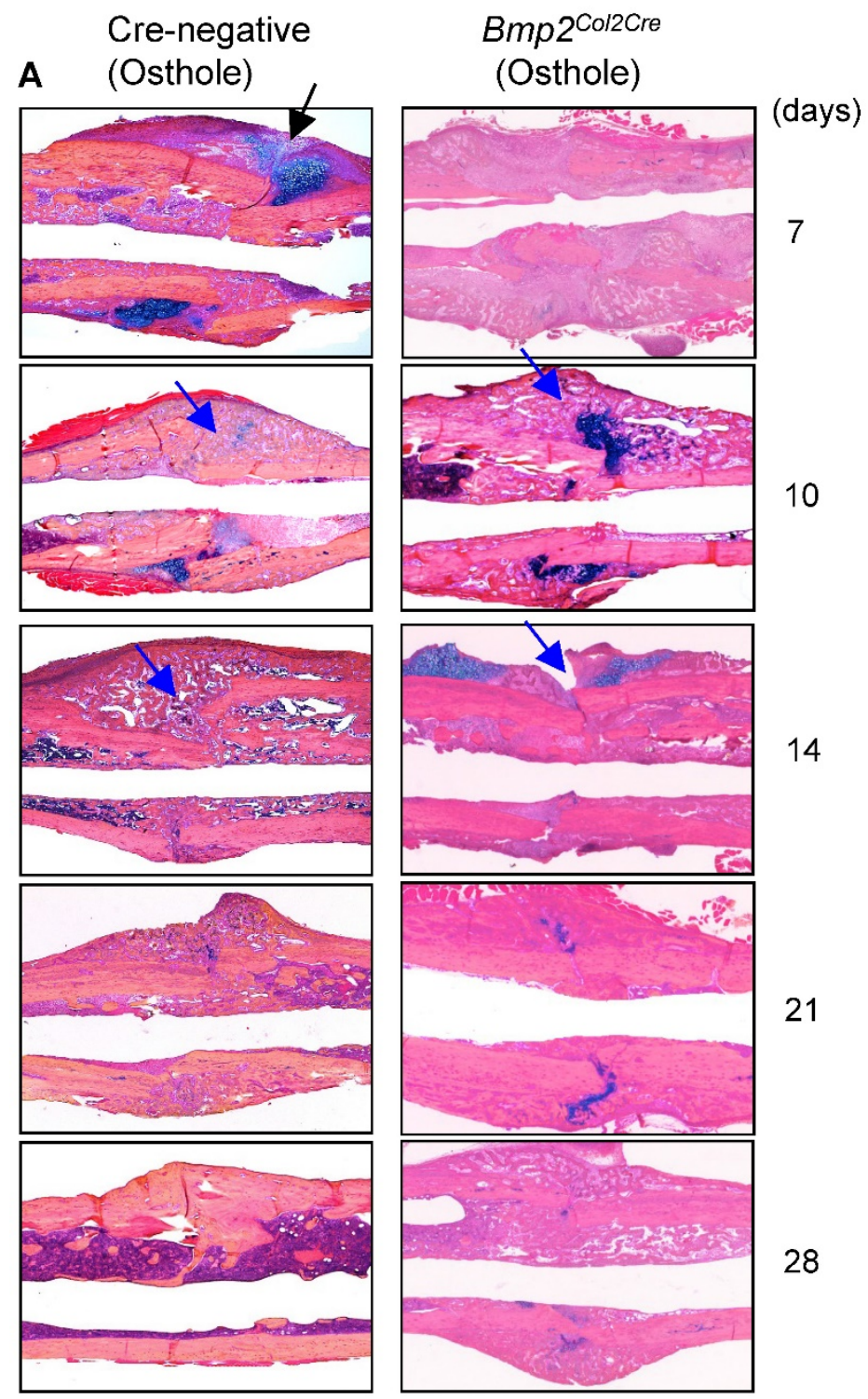

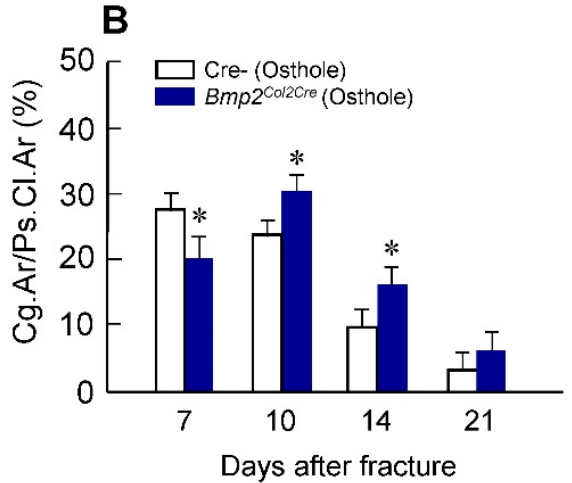

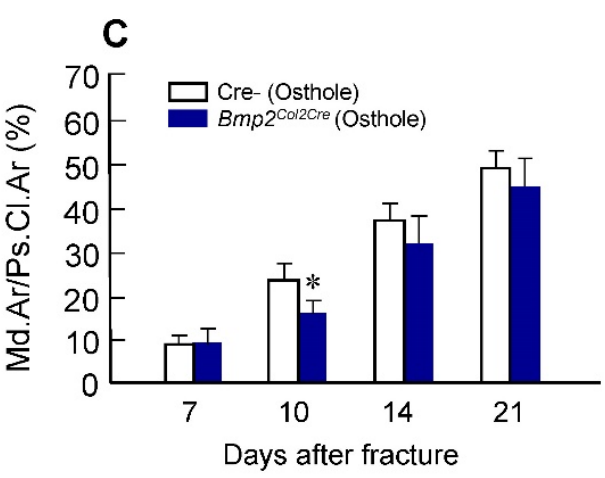

Figure 9. The effects of Osthole on the increases in cartilage and bone volume in fracture callus were inhibited Bmp2Col2Cre KO mice. (A) Histological analysis showed that Osthole could not accelerate the fracture healing process for Bmp2 Col2 Cre group. (B) The Alcian blue staining positive cartilage area of callus tissues (\%, Cg.Ar/Ps.Cl.Ar) was lower in Bmp2Col2Cre group compared with Cre-negative group after Osthole treatment at day 7. (C) New woven bone formation in callus tissues (\%, Md.Ar/Ps.Cl.Ar) was lower in Bmp2 Col2Cre group compared with Cre-negative group after Osthole treatment at day 10. Black arrows: cartilage area; blue arrows: woven bone area. Data are presented as means \pm SD. $* p<0.05, \mathrm{n}=10$. 

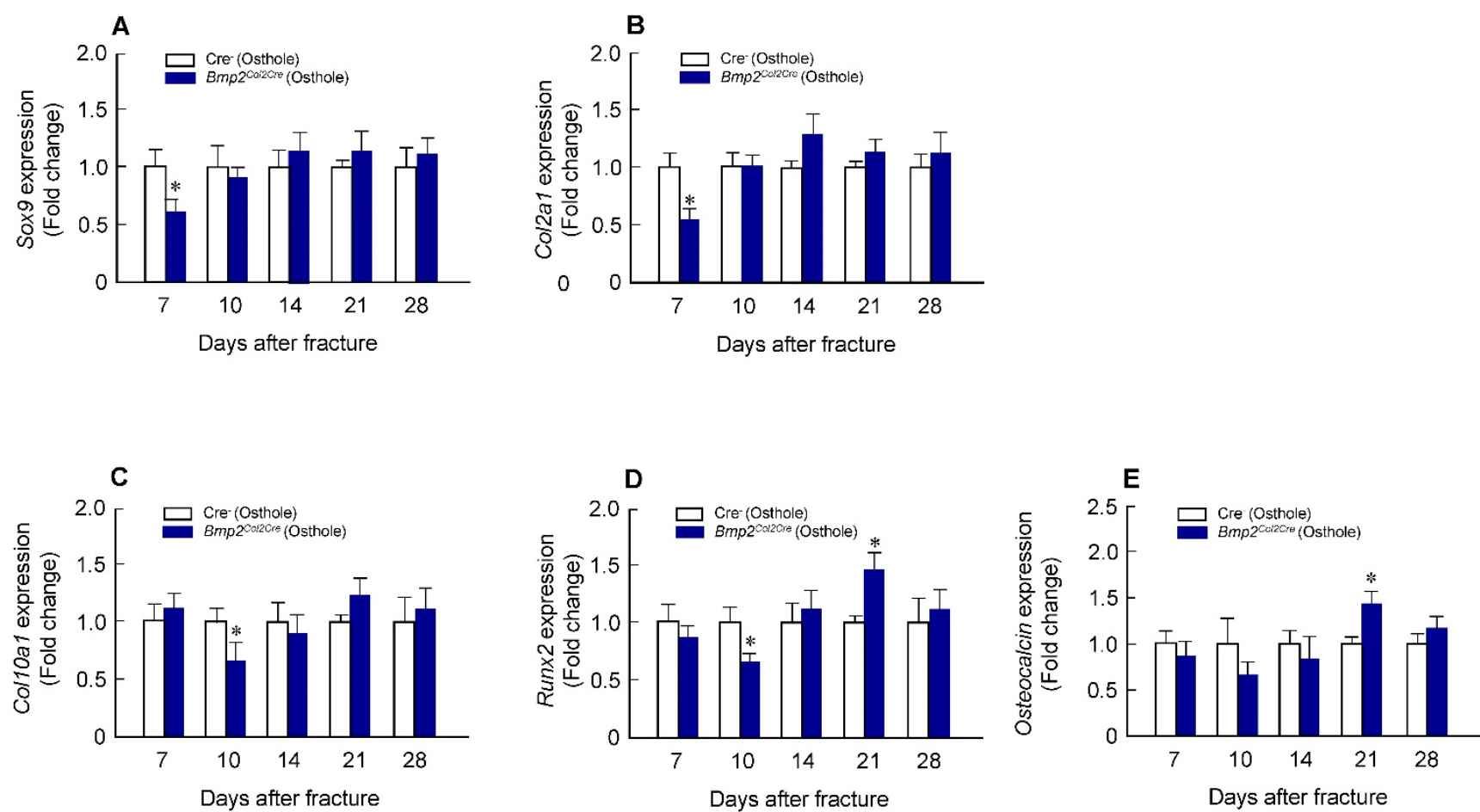

Figure 10. Osthole-induced chondrocyte and osteoblast gene expression was also inhibited in Bmp2Col2cre KO mice. (A-C) Expression of chondrocyte-specific genes, such as Sox9 (day 7), Col2al (day 7) and Coll Oal (day 10), was significantly lower in Bmp2Col2Cre group compared with Cre-negative control group. (D) Expression of osteoblast-specific genes, such as Runx2 (day 10), was also significantly lower in Bmp2Col2Cre group compared with Cre-negative control group. Data are presented as means \pm SD. $* p<0.05$, $n=6$.

\section{Discussion}

Osthole is a coumarin derivative that is present in many plants. These plants such as Cnidium monnieri and Angelica pubescens have been used as tonics and for the treatment of bone-related diseases $[13,30]$. Osthole stimulates bone formation both in vitro and in vivo [11, 12]. In the present study, we demonstrated that Osthole promotes bone fracture healing and its effect on fracture healing could be inhibited when Bmp2 was specifically deleted in chondrocytes. The results of the current study have identified the potential utility of Osthole in acceleration of fracture healing. The mechanism of Osthole-induced fracture healing may be partially related its role in activation of BMP signaling in chondrocytes.

Fracture healing can be temporally divided into an inflammatory phase, a soft callus phase, a hard callus phase, and a remodeling phase, and these stages show some overlap [31]. The bone repair process involves the coordination of multiple cell types to recover normal bone shape and function. Our data reveal that treatment with Osthole significantly accelerates the endochondral ossification in a fracture callus. Radiographic analysis showed that bridging of the fracture gap occurred at day 10 in Osthole-treated WT and Cre- control mice, which was evidently earlier than that observed in Bmp2Col2Cre mice. Bone volume was significantly increased in Osthole-treated group, compared to the vehicle control group and Bmp2Col2Cre $\mathrm{KO}$ group at days 7, 10 and 14. These data are consistent with histologic and histomorphometric analyse, showing that Osthole enhances fracture healing at the early chondrogenesis phase. Osthole-treated mice exhibited a fracture callus with a larger cartilaginous compartment compared to control group and Bmp2 $2^{\mathrm{Col} 2 \mathrm{Cre}} \mathrm{KO}$ group at day 7 . The increased chondrogenic response was associated with increased gene expression of Col2a1 and the protein expression of collagen II, a marker of cartilage formation at day 7. Sox 9 has been used as a marker for chondrogenic differentiation [32, 33] and it was upregulated at day 7 in Osthole-treated group. However, treatment with Osthole did not affect the expression of Col2a1 and Sox9 in Bmp2Col2Cre KO mice at the same time point. Col10a1 is expressed in hypertrophic chondrocytes which could further undergo mineralization and apoptosis, connecting to subsequent angiogenesis and endochondral bone formation [34, 35]. At day 10, the expression of Col10a1 was increased in Osthole-treated group, but not in Bmp2 Col2Cre group.

We then analyzed expression levels of osteogenic marker genes, such as Runx2 and osteocalcin. Runx2 is a transcription factor playing important roles in chondrocytes and osteoblasts. Osteocalcin is mainly expressed in mature osteoblasts 
although it is also detected in hypertrophic chondrocytes. The expression of Runx2 and osteocalcin was increased at day 21 when mice were treated with Osthole. Interestingly, between the two groups with Osthole treatment, the relative level of Runx2 mRNA in $\mathrm{Cre}^{-}$control group were significantly up-regulated than that of Bmp2Col2Cre group at day 10. Besides we utilized biomechanical testing and assessed maximal torque, stiffness and toughness. Stiffness reflects the elasticity of bone while maximal torque and toughness represent the forces and energy required to disrupt the callus. In our study, the results of maximal torque, stiffness and toughness reveal that Osthole enhances the mechanical strength of the bone fracture healing.

The findings presented above are consistent with our hypothesis that Osthole plays an important role during fracture healing by accelerating endochondral bone formation, especially during the chondrocyte differentiation and maturation. BMP2 plays an important role in chondrocyte differentiation and maturation [21]. BMP signaling pathway is known to regulate development and regeneration of bone and cartilage. BMPs elicit their function by binding to specific cell surface receptors with serine-threonine kinase activity and then inducing phosphorylation of receptor-regulated Smads (Smad1, 5, and 8) [36]. Smad1/5/8 specifically transduces BMP signal, overexpression of Smad1/5/8 in chondrocytes promotes chondrocyte maturation. Maturation of chondrocytes is a marked step in ossification. BMP2 promotes Smad1/5/8 phosphorylation and mediates Runx2 expression [37]. In our studies, the expression of pSmad1/5/8 was increased at day7 in Osthole-treated group, suggesting that Osthole promotes fracture healing through BMP2 signaling.

Therefore, we concluded that Osthole plays an important role in chondrocyte differentiation and maturation by regulating BMP2 signaling, and then influence endochondral ossification, thereby accelerating fracture healing.

\section{Abbreviations}

BMP: bone morphogenetic protein; IHC: immunohistochemistry; KO: knockout; WT: wild-type; DMSO: dimethylsulfoxide; OCN: osteocalcin.

\section{Acknowledgements}

This research has been partially supported by Zhejiang grants funded by Provincial Natural Science Foundation of China (Grant no. LY16H270010, LZ12H27001 and 81373669), the State Administration of Traditional Chinese Medicine of Zhejiang Province
(Grant no. 2016ZA048), China Postdoctoral Science Foundation (Grant no. 2016M590533) and the Program for Zhejiang Leading Team of S\&T Innovation and Key Laboratory of Zhejiang Province.

\section{Competing Interests}

The authors have declared that no competing interest exists.

\section{References}

1. Morgan EF, De Giacomo A, Gerstenfeld LC. Overview of skeletal repair (fracture healing and its assessment). Methods in molecular biology (Clifton, NJ). 2014; 1130: 13-31.

2. Lloret A, Coiffier G, Couchouron T, Perdriger A, Guggenbuhl P. Risk factors of mortality during the first year after low energy osteoporosis fracture: a retrospective case-control study. Clinical cases in mineral and bone metabolism 2016; 13: 123-6.

3. Griffin XL, Parsons N, Costa ML, Metcalfe D. Ultrasound and shockwave therapy for acute fractures in adults. The Cochrane database of systematic reviews. 2014:: Cd008579.

4. Gomez-Barrena E, Rosset P, Lozano D, Stanovici J, Ermthaller C, Gerbhard F. Bone fracture healing: cell therapy in delayed unions and nonunions. Bone. 2015; 70: 93-101.

5. Hadjiargyrou M, O'Keefe RJ. The convergence of fracture repair and stem cells: interplay of genes, aging, environmental factors and disease. Journal of bone and mineral research 2014; 29: 2307-22

6. Ebraheim NA, Buchanan GS, Liu X, Cooper ME, Peters N, Hessey JA, et al. Treatment of Distal Femur Nonunion Following Initial Fixation with a Lateral Locking Plate. Orthopaedic surgery. 2016; 8: 323-30.

7. Yao L, Lu P, Li Y, Yang L, Feng H, Huang Y, et al. Osthole relaxes pulmonary arteries through endothelial phosphatidylinositol 3-kinase/Akt-eNOS-NO signaling pathway in rats. European journal of pharmacology. 2013; 699: 23-32.

8. Hua KF, Yang SM, Kao TY, Chang JM, Chen HL, Tsai YJ, et al. Osthole mitigates progressive IgA nephropathy by inhibiting reactive oxygen species generation and NF-kappaB/NLRP3 pathway. PloS one. 2013; 8: e77794.

9. Alabi OD, Gunnink SM, Kuiper BD, Kerk SA, Braun E, Louters LL. Osthole activates glucose uptake but blocks full activation in L929 fibroblast cells, and inhibits uptake in HCLE cells. Life sciences. 2014; 102: 105-10.

10. Shi J, Fu Q, Chen W, Yang HP, Liu J, Wang XM, et al. Comparative study of pharmacokinetics and tissue distribution of osthole in rats after oral administration of pure osthole and Libanotis buchtormensis supercritical extract. Journal of ethnopharmacology. 2013; 145: 25-31.

11. Tang DZ, Hou W, Zhou Q, Zhang M, Holz J, Sheu TJ, et al. Osthole stimulates osteoblast differentiation and bone formation by activation of beta-catenin-BMP signaling. Journal of bone and mineral research : the official journal of the American Society for Bone and Mineral Research. 2010; 25: 1234-45.

12. Li XX, Hara I, Matsumiya T. Effects of osthole on postmenopausal osteoporosis using ovariectomized rats; comparison to the effects of estradiol. Biological \& pharmaceutical bulletin. 2002; 25: 738-42.

13. Ko FN, Wu TS, Liou MJ, Huang TF, Teng CM. Vasorelaxation of rat thoracic aorta caused by osthole isolated from Angelica pubescens. European journal of pharmacology. 1992; 219: 29-34.

14. Vortkamp A, Pathi S, Peretti GM, Caruso EM, Zaleske DJ, Tabin CJ Recapitulation of signals regulating embryonic bone formation during postnatal growth and in fracture repair. Mechanisms of development. 1998; 71: 65-76.

15. Deschaseaux F, Sensebe L, Heymann D. Mechanisms of bone repair and regeneration. Trends in molecular medicine. 2009; 15: 417-29.

16. Simic P, Vukicevic S. Bone morphogenetic proteins: from developmental signals to tissue regeneration. Conference on bone morphogenetic proteins. EMBO reports. 2007; 8: 327-31.

17. Govender S, Csimma C, Genant HK, Valentin-Opran A, Amit Y, Arbel R, et al. Recombinant human bone morphogenetic protein-2 for treatment of open tibial fractures: a prospective, controlled, randomized study of four hundred and fifty patients. The Journal of bone and joint surgery American volume. 2002; 84a: 2123-34.

18. Ming LG, Zhou J, Cheng GZ, Ma HP, Chen KM. Osthol, a coumarin isolated from common cnidium fruit, enhances the differentiation and maturation of osteoblasts in vitro. Pharmacology. 2011; 88: 33-43.

19. Kuo PL, Hsu YL, Chang $\mathrm{CH}$, Chang JK. Osthole-mediated cell differentiation through bone morphogenetic protein-2/p38 and extracellular signal-regulated kinase $1 / 2$ pathway in human osteoblast cells. The Journal of pharmacology and experimental therapeutics. 2005; 314: 1290-9.

20. Zhang Z, Leung WN, Li G, Lai YM, Chan CW. Osthole Promotes Endochondral Ossification and Accelerates Fracture Healing in Mice. Calcified tissue international. 2016; 99: 649-60. 
21. Shu B, Zhang M, Xie R, Wang M, Jin H, Hou W, et al. BMP2, but not BMP4, is crucial for chondrocyte proliferation and maturation during endochondral bone development. Journal of cell science. 2011; 124: 3428-40.

22. Mi M, Jin H, Wang B, Yukata $\mathrm{K}$, Sheu TJ, Ke QH, et al. Chondrocyte BMP2 signaling plays an essential role in bone fracture healing. Gene. 2013; 512: 211-8.

23. Dacquin R, Starbuck M, Schinke T, Karsenty G. Mouse alpha1(I)-collagen promoter is the best known promoter to drive efficient Cre recombinase expression in osteoblast. Developmental dynamics 2002; 224: 245-51.

24. Ovchinnikov VA, Men'kov AV. [Choice of surgical treatment method for peptic ulcer of operated stomach]. Khirurgiia. 2000: 15-8.

25. Schindeler A, Morse A, Harry L, Godfrey C, Mikulec K, McDonald M, et al. Models of tibial fracture healing in normal and Nf1-deficient mice. Journal of orthopaedic research 2008; 26: 1053-60.

26. Jin H, Wang B, Li J, Xie W, Mao Q, Li S, et al. Anti-DKK1 antibody promotes bone fracture healing through activation of beta-catenin signaling. Bone. 2015; 71: 63-75.

27. O'Neill KR, Stutz CM, Mignemi NA, Burns MC, Murry MR, Nyman JS, et al. Micro-computed tomography assessment of the progression of fracture healing in mice. Bone. 2012; 50: 1357-67.

28. Dai F, Lin X, Chang C, Feng XH. Nuclear export of Smad2 and Smad3 by RanBP3 facilitates termination of TGF-beta signaling. Developmental cell. 2009; 16: 345-57.

29. Gao Z, Wen $\mathrm{O}$, Xia $Y$, Yang I, Gao P, Zhang $N$, et al. Osthole augments therapeutic efficiency of neural stem cells-based therapy in experimental autoimmune encephalomyelitis. Journal of pharmacological sciences. 2014; 124: 54-65.

30. Teng CM, Lin $\mathrm{CH}, \mathrm{Ko} F N$, Wu TS, Huang TF. The relaxant action of osthole isolated from Angelica pubescens in guinea-pig trachea. Naunyn-Schmiedeberg's archives of pharmacology. 1994; 349: 202-8.

31. Schindeler A, McDonald MM, Bokko P, Little DG. Bone remodeling during fracture repair: The cellular picture. Seminars in cell \& developmental biology. 2008; 19: 459-66.

32. Lefebvre V, Huang W, Harley VR, Goodfellow PN, de Crombrugghe B. SOX9 is a potent activator of the chondrocyte-specific enhancer of the pro alpha1(II) collagen gene. Molecular and cellular biology. 1997; 17: 2336-46.

33. Bridgewater LC, Lefebvre V, de Crombrugghe B. Chondrocyte-specific enhancer elements in the Col11a2 gene resemble the Col2a1 tissue-specific enhancer. The Journal of biological chemistry. 1998; 273: 14998-5006.

34. Kugimiya F, Kawaguchi H, Kamekura S, Chikuda H, Ohba S, Yano F, et al. Involvement of endogenous bone morphogenetic protein (BMP) 2 and BMP6 in bone formation. The Journal of biological chemistry. 2005; 280: 35704-12.

35. Chung UI, Schipani E, McMahon AP, Kronenberg HM. Indian hedgehog couples chondrogenesis to osteogenesis in endochondral bone development. The Journal of clinical investigation. 2001; 107: 295-304.

36. Wan M, Cao X. BMP signaling in skeletal development. Biochemical and biophysical research communications. 2005; 328: 651-7.

37. Sheu TI, Zhou W, Fan J, Zhou H, Zuscik MJ, Xie C, et al. Decreased BMP2 signal in GIT1 knockout mice slows bone healing. Molecular and cellular biochemistry. 2014; 397: 67-74. 